九州大学学術情報リポジトリ

Kyushu University Institutional Repository

\title{
ESTIMATION OF VARIANCE AFTER PRELIMINARY CONJECTURE
}

Inada, Koichi

Department of Mathematics, Faculty of Science, Kagoshima University

https://doi.org/10.5109/13405

出版情報: Bulletin of informatics and cybernetics. 23 (3/4)，pp.183-198，1989-03. Research Association of Statistical Sciences

バージョン :

権利関係 : 


\title{
ESTIMATION OF VARIANCE AFTER PRELIMINARY CONJECTURE
}

\section{By}

\author{
Koichi INADA*
}

\begin{abstract}
In estimating parameter of the underlying distribution, the statistician may have prior information about parameter such that the true value of parameter lies in the neighborhood of a known value. We consider the problem of estimating variance of normal distribution when it is preliminarily conjectured that the true of variance lies in the interval. We suggest two estimators and compare these two estimators with the preliminary test estimator and the sample unbiased variance by using the mean square error.
\end{abstract}

\section{Introduction}

The classical statistical inferences utilizes only the sample information which is obtained by the statistical investigation to make inference about unknown parameter of the underlying distribution. That is, it disregard the situation to which it is put.

On the other hand, we shall take a stand which utilizes not only the sample information but also other relevant informations to making the suitable decision. (We shall explain our standpoint and method of inference, by quoting Berger [7]).

There are two types of such information. The one is a knowledge of the con sequences of each possible inferences and is described by the loss that will occur for each inferences and possible values of parameter. The notion of loss is very important problem in statistical inference. But we do not concern ourself in this respect and we take the usual squared loss as the loss function.

The other is prior information which yields some information for the underlying distribution without sample. The one approach to statistical inference which makes use of such prior information is Bayesian analysis. In this approach unknown parameter is considered random variable and prior information is described by a probability dis tribution of the parameter, which is called prior distribution.

In this paper we do not take the Bayesian standpoint and take the following approach to statistical inference. The underlying distributions are assumed to have known types of distribution and to include unknown parameters. Prior information about parameters is vaguely given by information that true parameters will have some property. The one method of statistical inference using such prior information is the well-known estimation after preliminary test. The idea of this estimation method was

\footnotetext{
* Department of Mathematics, Faculty of Science, Kagoshima University, Kagoshima 890, Japan.
} 
first introduced by Bancroft [4]. On the basis of the preliminary test, for example, the decision is made whether to pool two samples or to use only one sample. We consider estimation of mean $\mu_{1}$ of normal distribution $N\left(\mu_{1}, \sigma_{1}^{2}\right)$. We have the one sample from $N\left(\mu_{1}, \sigma_{1}^{2}\right)$ and the other sample from $N\left(\mu_{2}, \sigma_{2}^{2}\right)$. Available prior information is that $\mu_{2}$ will be close to $\mu_{1}$. If the difference of the two sample means derived from the above samples is small, then we use the two samples to estimate $\mu_{1}$. Otherwise, we utilize only the sample from $N\left(\mu_{1}, \sigma_{1}^{2}\right)$ to estimate $\mu_{1}$. Further investigations of the estimation after preliminary test are worked by Kitagawa [12], Bennett [6] and Asano [1], [2], [3]. A bibliograhy in Bancroft and Han [5] is a good source of references. The preliminary test estimator always depends on the level of significance of the preliminary test. Hirano [8], [9] discussed the level of significance of the preliminary test for the mean of the normal distribution. Similarly, Toyoda and Wallace [15], Ohtani and Toyoda [14] and Hirano [10], [11] discussed the level of significance of the preliminary test for the variance of the normal distribution.

On the other hand, different from estimation after preliminary test, we shall consider a method of estimation by making use of the prior information represented such that true parameter lies in an interval as preliminary conjecture. Thus our method of estimation is considered as estimation after preliminary conjecture. In this paper we shall consider the estimation of variance of the normal distribution under the above situation.

Let $X_{1}, X_{2}, \cdots, X_{n}$ be a sample of size $n$ from a normal distribution with unknown mean $\mu$ and unknown variance $\sigma^{2}$. We shall consider the estimation problem of unknown variance $\sigma^{2}$. The well-known estimator of $\sigma^{2}$ is the sample unbiased variance $U$ given by

$$
U=\sum_{i=1}^{n}\left(X_{i}-\bar{X}\right)^{2} /(n-1),
$$

where $\bar{X}$ is a sample mean.

As previously stated, there is often some available prior information about $\sigma^{2}$. Under such situations the statistician should utilize that information to estimate $\sigma^{2}$.

Suppose that we have prior information such that $\sigma^{2}$ is close to a known positive value $\sigma_{0}^{2}$.

The one method to utilize this information is estimation after preliminary test. At first we consider to test the hypothesis $H_{0}: \sigma^{2}=\sigma_{0}^{2}$. If the hypothesis is not rejected, then we estimate $\sigma^{2}$ by $\sigma_{0}^{2}$. If the hypothesis is rejected, then we estimate $\sigma^{2}$ by the sample unbiased variance $U$. This leads to the preliminary test estimator given by

$$
P T= \begin{cases}\sigma_{0}^{2} & \text { if } \chi_{\bar{n}-1}^{2}(1-\alpha / 2) /(n-1)<U / \sigma_{0}^{2}<\chi_{n-1}^{2}(\alpha / 2) /(n-1), \\ U & \text { otherwise, }\end{cases}
$$

where $\chi_{n-1}^{2}(\alpha)$ is the upper $100 \%$ point of the chi-square distribtion with $n-1$ degrees of freedom.

Different from the above estimation after preliminary test, in the meantime, we shall represent the prior information as that the true value of $\sigma^{2}$ lies in the some interval containing a known value $\sigma_{0}^{2}$. That is, we consider the situation such that we can preliminarily conjecture that the true value of variance lies in the interval 
$\left[\sigma_{0}^{2} / C_{0}, \sigma_{0}^{2} C_{0}\right]$, where $C_{0}$ is a positive constant. An important problem resulted from the above preliminary conjecture is how one incorporate this prior informations in the estimation of $\sigma^{2}$.

In Section 2, we propose two types of estimators of variance of the normal distribution, which utilize the preliminary conjecture. We also give their mean square errors.

In Section 3, based on the mean square error criterion, we make comparison among estimators, which are the two estimators proposed in Section 2, the preliminary test estimator and the sample unbiased variance.

\section{Estimation after Preliminary Conjecture}

Let $X_{1}, X_{2}, \cdots, X_{n}$ be a sample of size $n$ from a normal distribution with unknown mean $\mu$ and unknown variance $\sigma^{2}$. We have prior information that the true value of $\sigma^{2}$ lies in the interval $\left[\sigma_{0}^{2} / C_{0}, \sigma_{0}^{2} C_{0}\right]$, where $\sigma_{0}^{2}$ and $C_{0}$ are known positive constants. Utilizing this prior information, we shall propose two types of estimators of variance $\sigma^{2}$. For each type, we shall determine the optimal estimator by the minimax criterion. Type I:

We consider the following type of estimator;

$$
T(w, C)=\left\{\begin{array}{lll}
\sigma_{0}^{2} & \text { if } & C^{-1}<U / \sigma_{0}^{2}<C \\
w U & \text { if } & U / \sigma_{0}^{2} \geqq C \\
w^{-1} U & \text { if } & U / \sigma_{0}^{2} \leqq C^{-1}
\end{array}\right.
$$

where $U=\sum_{i=1}^{n}\left(X_{i}-\bar{X}\right)^{2} /(n-1)$, the weight $w$ is a constant such that $0<w \leqq 1$ and $C$ is a positive constant.

Let $\operatorname{MSE}[T(w, C)]$ be a mean square error of the estimator $T(w, C)$, i. e., $\operatorname{MSE}[T(w, C)]=E\left[T(w, C)-\sigma^{2}\right]^{2}$. We shall determine the weight $w$ and $C$ by the minimax criterion. That is, we shall determine the optimal estimator $T\left(w_{1}, C_{1}\right)$ with $w_{1}$ and $C_{1}$ which satisfys

$$
\operatorname{MSE}\left[T\left(w_{1}, C_{1}\right)\right]=\operatorname{Inf}_{0<w \leqq 1, C>0} \operatorname{Sup}_{\sigma^{2} \in\left[\sigma_{0}^{2} / C_{0}, \sigma_{0}^{2} C_{0}\right]} \operatorname{MSE}[T(w, C)]
$$

where $T(w, C)$ is given by (2.1). Thus the estimator $T\left(w_{1}, C_{1}\right)$ is the minimax estimator.

In the meantime, we can also consider

$$
\operatorname{Sup}_{\sigma^{2}=\left[\sigma_{0}^{2} / C_{0}, \sigma_{0}^{2} C_{0}\right]} \operatorname{MSE}[T(w, C)]
$$

as the mean-max risk of Kudo [13], where we use the prior information that $\sigma^{2}$ lies in the interval $\left[\sigma_{0}^{2} / C_{0}, \sigma_{0}^{2} C_{0}\right]$ with probability one. In this regard, the minimax estimator $T\left(w_{1}, C_{1}\right)$ is considered the estimator which minimizes the mean-max risk

$$
\operatorname{Sup}_{\sigma^{2} \in\left[\sigma_{0}^{2} / C_{0}, \sigma_{0}^{2} C_{0}\right]} \operatorname{MSE}[T(w, C)] \quad \text { for } 0<w \leqq 1 \text { and } C>0,
$$

among the class of estimators given by (2.1).

Hereafter we shall denote the estimator $T\left(w_{1}, C_{1}\right)$ by $T 1$. That is, the estimator 
$T 1$ is given by

$$
T 1=\left\{\begin{array}{lll}
\sigma_{0}^{3} & \text { if } \quad C_{1}^{-1}<U / \sigma_{0}^{2}<C_{1} \\
w_{1} U & \text { if } \quad U / \sigma_{0}^{2} \geqq C_{1} \\
w_{1}^{-1} U & \text { if } & U / \sigma_{0}^{2} \leqq C_{1}^{-1}
\end{array}\right.
$$

where the weight $w_{1}$ and $C_{1}$ are determined to satisfy (2.2) with given values $\sigma_{0}^{2}$ and $C_{0}$.

Type II:

We consider the following type of estimator;

$$
T(w)=\left\{\begin{array}{lll}
w U & \text { if } & U / \sigma_{0}^{2}>1 \\
w^{-1} U & \text { if } & U / \sigma_{0}^{2} \leqq 1
\end{array}\right.
$$

where the weight $w$ is a constant such that $0<w \leqq 1$. We shall determine the weight $w$ by the minimax criterion. That is, we shall determine the optimal estimator $T\left(w_{2}\right)$ with $w_{2}$ which satisfys

$$
\operatorname{MSE}\left[T\left(w_{2}\right)\right]=\operatorname{Inf}_{0<w \leq 1} \operatorname{Sup}_{\sigma^{2}=\left[\sigma_{0}^{2} / C_{0}, \sigma_{0}^{2} C_{0}\right]} \operatorname{MSE}[T(w)]
$$

where $T(w)$ is given by (2.4). Thus the estimator $T\left(w_{2}\right)$ is the minimax estimator. Similar to the estimator $T 1, T 2$ is also considered the estimator which minimizes the mean-max risk among the class of estimators given by (2.4). Hereafter $T\left(w_{2}\right)$ is denoted by $T 2$. That is, the estimator $T 2$ is given by

$$
T 2=\left\{\begin{array}{lll}
w_{2} U & \text { if } & U / \sigma_{0}^{2}>1 \\
w_{2}^{-1} U & \text { if } & U / \sigma_{0}^{2} \leqq 1
\end{array}\right.
$$

where the weight $w_{2}$ is determined to satisfy (2.5) with given values $\sigma_{0}^{2}$ and $C_{0}$.

We evaluate the mean square errors of the estimators given by $(2.1)$ and (2.4) in the following Theorem and its corollary.

THEOREM. Let $X_{1}, X_{2}, \cdots, X_{n}$ be a sample of size $n$ from a normal distribution with unknown mean $\mu$ and unknown variance $\sigma^{2}$. Then the mean square error of the estimator given by (2.1) is

$$
\begin{aligned}
\operatorname{MSE}[T(w, C)]= & \sigma^{4}\left[\left\{w^{-2} I_{a}\left(\beta_{1}\right)+w^{2}\left(1-I_{b}\left(\beta_{1}\right)\right)\right\}(n+1) /(n-1)\right. \\
& \left.-2\left\{w^{-1} I_{a}\left(\beta_{2}\right)+w\left(1-I_{b}\left(\beta_{2}\right)\right)\right\}+1\right] \\
& +\left(2 \sigma_{0}^{2} \sigma^{2}-\sigma_{0}^{4}\right)\left\{I_{a}\left(\beta_{3}\right)-I_{b}\left(\beta_{3}\right)\right\}
\end{aligned}
$$

where $I_{t}(\beta)$ is an incomplete gamma function ratio, that is,

$$
\begin{gathered}
I_{t}(\beta)=\Gamma(\beta)^{-1} \int_{0}^{t} z^{\beta-1} e^{-z} d z, \beta_{1}=(n+3) / 2, \beta_{2}=(n+1) / 2, \\
\beta_{3}=(n-1) / 2, a=(n-1) C^{-1} \sigma_{0}^{2} /\left(2 \sigma^{2}\right) \text { and } b=(n-1) C \sigma_{0}^{2} /\left(2 \sigma^{2}\right) .
\end{gathered}
$$

Before the proof of Theorem, we shall prepare the following lemma.

LEMMA. Let $\chi^{2}$ be a random variable which have a chi-square distribution with $n-1$ degrees of freedom and $f\left(\chi^{2}\right)$ be its probability density function, that is, 
Then we have

$$
f\left(\chi^{2}\right)=\Gamma[(n-1) / 2]^{-1}\left(\chi^{2} / 2\right)^{(n-1) / 2} \exp \left[-\chi^{2} / 2\right]
$$

$$
\int_{t}^{\infty} f\left(\chi^{2}\right) d \chi^{2}=1-I_{t / 2}\left(\beta_{3}\right), \int_{t}^{\infty} \chi^{2} f\left(\chi^{2}\right) d \chi^{2}=(n-1)\left\{1-I_{t / 2}\left(\beta_{2}\right)\right\}
$$

and

$$
\int_{t}^{\infty}\left(\chi^{2}\right)^{2} f\left(\chi^{2}\right) d \chi^{2}=(n-1)(n+1)\left\{1-I_{t / 2}\left(\beta_{1}\right)\right\}
$$

This lemma can be proved by applying the transformation of variables, $z=\chi^{2} / 2$.

Proof of THEOREM. For the estimator $T(w, C)$ given by $(2.1)$, its mean square error is given by

$$
\begin{aligned}
\operatorname{MSE}[T(w, C)]= & \int_{C^{-1} \sigma_{0}^{2}}^{C \sigma_{0}^{2}}\left(\sigma_{0}^{2}-\sigma^{2}\right)^{2} g(u) d u+\int_{C \sigma_{0}^{2}}^{\infty}\left(u \cdot u-\sigma^{2}\right)^{2} g(u) d u \\
& +\int_{0}^{C-1 \sigma_{0}^{2}}\left(w^{-1} u-\sigma^{2}\right)^{2} g(u) d u
\end{aligned}
$$

where $g(u)=\frac{(n-1)}{\sigma^{2}} \Gamma\left(\frac{n-1}{2}\right)^{-1}\left[\frac{(n-1) u}{2 \sigma^{2}}\right]^{n-1} \exp \left[-\frac{(n-1) u}{2 \sigma^{2}}\right]$.

Making a transformation $\chi^{2}=(n-1) u / \sigma^{2}$ and putting $a=(n-1) C^{-1} \sigma_{0}^{2} /\left(2 \sigma^{2}\right)$ and $b=$ $(n-1) C \sigma_{0}^{2} /\left(2 \sigma^{2}\right)$, we have

$$
\begin{aligned}
\operatorname{MSE}[T(w, C)]= & \int_{2 a}^{2 b}\left(\sigma_{0}^{2}-\sigma^{2}\right) f\left(\chi^{2}\right) d \chi^{2}+\int_{2 b}^{\infty}\left(w \sigma^{2} \chi^{2} /(n-1)-\sigma^{2}\right)^{2} \\
& f\left(\chi^{2}\right) d \chi^{2}+\int_{0}^{2 a}\left(w^{-1} \sigma^{2} \chi^{2} /(n-1)-\sigma^{2}\right)^{2} f\left(\chi^{2}\right) d \chi^{2} \\
= & \left(\sigma_{0}^{2}-\sigma^{2}\right)^{2} \int_{2 a}^{2 b} f\left(\chi^{2}\right) d \chi^{2}+\sigma^{4}(n-1)^{-2}\left[\int _ { 2 b } ^ { \infty } \left\{w^{2}\left(\chi^{2}\right)^{2}\right.\right. \\
& \left.-2 w(n-1) \chi^{2}+(n-1)^{2}\right\} f\left(\chi^{2}\right) d \chi^{2}+\int_{0}^{2 a}\left\{u^{-2}\left(\chi^{2}\right)^{2}\right. \\
& \left.\left.-2 w^{-1}(n-1) \chi^{2}+(n-1)^{2}\right\} f\left(\chi^{2}\right) d \chi^{2}\right] .
\end{aligned}
$$

By applying Lemma to the above equation, we have the mean square error $\operatorname{MSE}[T(u, C)]$ given by (2.7).

Specially when $C=1$, the estimator $T(w, C)$ given by $(2.1)$ is reduced to the estimator $T(w)$ given by (2.4). Thus we have the following corollary.

COROLLARY 1. The mean square error of the estimator given by (2.4) is

$$
\begin{aligned}
\operatorname{MSE}[T(w)]= & \sigma^{4}\left[\left\{w^{-2} I_{d}\left(\beta_{1}\right)+w^{2}\left(1-I_{d}\left(\beta_{1}\right)\right)\right\}(n+1) /(n-1)\right. \\
& \left.-2\left\{w^{-1} I_{d}\left(\beta_{2}\right)+w\left(1-I_{d}\left(\beta_{2}\right)\right)\right\}+1\right]
\end{aligned}
$$

where $d=(n-1) \sigma_{0}^{2} /\left(2 \sigma^{2}\right), \beta_{1}=(n+3) / 2$ and $\beta_{2}=(n+1) / 2$.

It is noted that an incomplete gamma function ratio $I_{t}(\beta)$ can be calculated in case of $\beta=\beta^{\prime} / 2$ where $\beta^{\prime}$ is a positive integer by using the following equation (see Yamauti [16], for example);

$$
I_{t}(\beta)=1-Q\left(2 \chi^{2} ; \beta^{\prime} / 2\right)
$$


where

when $\nu$ is even, $Q\left(\chi^{2} ; \nu\right)=e^{-\chi^{2} / 2}\left[1+\frac{\chi^{2}}{2}+\frac{\chi^{4}}{2 \cdot 4}+\cdots+\frac{\chi^{\nu-2}}{2 \cdot 4 \cdots \cdot(\nu-2)}\right]$

and

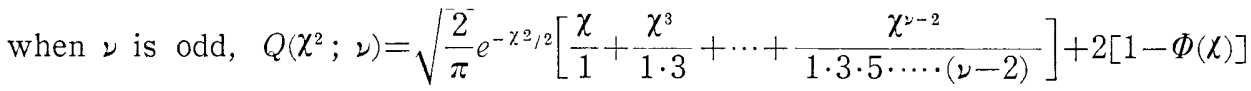

where $\Phi$ is the cumulative distribution function of the standard normal distribution function.

As it seems difficult to find $w_{1}$ and $C_{1}$ sayisfying the criterion (2.2) analytically as yet, we resort to numerical computations with a fixed sample size $n$. From numerical computations, it is seen that $\operatorname{MSE}[T(w, C)]$ for $\sigma^{2} \in\left[\sigma_{0}^{2} / C_{0}, \sigma_{0}^{2} C_{0}\right]$ attains a maximum at $\sigma^{2}=\sigma_{0}^{2} C_{0}$ for every fixed $w$ and $C$. We first calculate $\operatorname{MSE}[T(w, C)]$ at $\sigma^{2}=\sigma_{0}^{2} C_{0}$ for every fixed $w$ and $C$. Next we numerically evaluate values of $w$ and $C$ such that $w$ and $C$ attain a minimum of $\operatorname{Sup}_{\sigma^{2} \in\left[\sigma_{0}^{2} / C_{0}, \sigma_{0}^{2} C_{0}\right]} \operatorname{MSE}[T(w, C)]$ for $0<w \leqq 1$ and $C>0$. Similarly, we evaluate numerically a value of $w$ satisfying (2.5).

Numerical values of $w_{1}, C_{1}$ and $w_{2}$ are listed in Table 1 for $\sigma_{0}^{2}=1, C_{0}=1.5,2.0,2.5$ and some values of $n$.

From Table 1, it is clear that for each $n w_{1}$ and $w_{2}$ increase and $C_{1}$ decreases as $C_{0}$ increases, i. e., the range of the interval which is preliminarily conjectured increases.

For a fixed $C_{0}$ as $n$ increases, $w_{1}$ and $w_{2}$ increase and tend to be numerically almost equal and $C_{1}$ decreases to one. Therefore the estimator $T 1$ is almost indistinguishable from the estimator $T 2$ for large $n$.

Using the values of $w_{1}, C_{1}$ and $w_{2}$ given in Table 1 and Theorem, we evaluate

Table 1. Values of $w_{1}$ and $C_{1}$ of the minimax estimator $T 1$ and $w_{2}$ of the minimax estimator $T 2$.

\begin{tabular}{|c|c|c|c|c|c|c|c|c|c|}
\hline$n$ & $C_{0}$ & $w_{1}$ & $C_{1}$ & $w_{2}$ & $n$ & $C_{0}$ & $w_{1}$ & $C_{1}$ & $w_{2}$ \\
\hline \multirow{3}{*}{7} & 1.5 & .422 & 2.37 & .618 & \multirow{3}{*}{30} & 1.5 & .880 & 1.14 & .893 \\
\hline & 2.0 & .626 & 1.60 & .665 & & 2.0 & .929 & 1.08 & .930 \\
\hline & 2.5 & .686 & 1.46 & .701 & & 2.5 & .935 & 1.07 & .935 \\
\hline \multirow{3}{*}{10} & 1.5 & .591 & 1.69 & .698 & \multirow{3}{*}{35} & 1.5 & .903 & 1.11 & .911 \\
\hline & 2.0 & .739 & 1.35 & .760 & & 2.0 & .941 & 1.06 & .941 \\
\hline & 2.5 & .783 & 1.28 & .791 & & 2.5 & .944 & 1.06 & .944 \\
\hline \multirow{3}{*}{15} & 1.5 & .726 & 1.38 & .781 & \multirow{3}{*}{40} & 1.5 & .919 & 1.09 & .925 \\
\hline & 2.0 & .835 & 1.20 & .843 & & 2.0 & .949 & 1.06 & .949 \\
\hline & 2.5 & .862 & 1.16 & .864 & & 2.5 & .951 & 1.06 & .951 \\
\hline \multirow{3}{*}{20} & 1.5 & .801 & 1.25 & .833 & \multirow{3}{*}{50} & 1.5 & .942 & 1.06 & .944 \\
\hline & 2.0 & .884 & 1.13 & .887 & & 2.0 & .960 & 1.04 & .960 \\
\hline & 2.5 & .900 & 1.11 & .900 & & 2.5 & .961 & 1.04 & .961 \\
\hline \multirow{3}{*}{25} & 1.5 & .849 & 1.18 & .868 & \multirow{3}{*}{100} & 1.5 & .978 & 1.02 & .978 \\
\hline & 2.0 & .911 & 1.10 & .913 & & 2.0 & .980 & 1.01 & .980 \\
\hline & 2.5 & .921 & 1.09 & .921 & & 2.5 & .980 & 1.01 & .980 \\
\hline
\end{tabular}


numerically the mean square errors of the minimax estimators $T 1$ and $T 2$.

The mean square errors of the sample unbiased variance $U$ and the preliminary test estimator $P T$ given by (1.1) are given in the following proposition.

Proposition 1. Let $X_{1}, X_{2}, \cdots, X_{n}$ be a sample of size $n$ from a normal distribution $N\left(\mu, \sigma^{2}\right)$. Then the mean square errors of the sample unbiased variance $U$ and the preliminary test estimator $P T$ given by (1.1) are respectively,

and

$$
\operatorname{MSE}(U)=2 \sigma^{4} /(n-1)
$$

$$
\begin{aligned}
\operatorname{MSE}(P T)= & \sigma^{4}\left[\left\{I_{e}\left(\beta_{1}\right)-I_{f}\left(\beta_{1}\right)+1\right\}(n+1) /(n-1)-2\left\{I_{e}\left(\beta_{2}\right)-I_{f}\left(\beta_{2}\right)\right\}-1\right] \\
& +\left(2 \sigma_{0}^{2} \sigma^{2}-\sigma_{0}^{4}\right)\left\{I_{e}\left(\beta_{3}\right)-I_{f}\left(\beta_{3}\right)\right\}
\end{aligned}
$$

where $e=\chi_{n-1}^{2}(1-\alpha / 2) \sigma_{0}^{2} /\left(2 \sigma^{2}\right)$ and $f=\chi_{n-1}^{2}(\alpha / 2) \sigma_{0}^{2} /\left(2 \sigma^{2}\right)$.

\section{Comparison of Estimators}

Let $X_{1}, X_{2}, \cdots, X_{n}$ be a sample of size $n$ from a normal distribution $N\left(\mu, \sigma^{2}\right)$. Prior information is that $\sigma^{2}$ lies in the interval $\left[\sigma_{0}^{2} / C_{0}, \sigma_{0}^{2} C_{0}\right]$, where $\sigma_{0}^{2}=1$ and $C_{0}=$ $1.5,2.0$ or 2.5. Note that we can assume $\sigma_{0}^{2}=1$ without loss of generality. To make a comparison among four estimators which are the minimax estimators $T 1, T 2$, the preliminary test estimator $P T$ and the sample unbiased variance $U$, we shall use the ratios of the mean square errors of $T 1, T 2$ and $P T$ to the one of $U$ which is the uniformly minimum variance unbiased estimator of $\sigma^{2}$. The above ratio is also called efficiency. We shall denote the efficiencies of $T 1, T 2$ and $P T$ relative to $U$ by $e(T 1)$, $e(T 2)$ and $e(P T)$, respectively. That is,

$$
\begin{aligned}
& e(T 1)=\operatorname{MSE}(U) / \operatorname{MSE}(T 1), \\
& e(T 2)=\operatorname{MSE}(U) / \operatorname{MSE}(T 2), \\
& e(P T)=\operatorname{MSE}(U) / \operatorname{MSE}(P T),
\end{aligned}
$$

which depend on $\sigma^{2}$.

If $e(T 1)>1$, then $T 1$ is more efficient than $U$, and if $e(T 1)>e(T 2)$, then $T 1$ is more efficient than $T 2$ in the sense of the mean square error.

As $\sigma^{2}$ tends to infinity, we have easily the following properties of the efficiencies by Theorem and Proposition 1 .

Proposition 2. As $\sigma^{2}$ tends to infinity, we have

$$
\begin{aligned}
& \lim _{\sigma^{2} \rightarrow \infty} e(T 1)=2 /\left\{(n+1) w_{1}^{2}-2(n-1) w_{1}+n-1\right\}, \\
& \lim _{\sigma^{2} \rightarrow \infty} e(T 2)=2 /\left\{(n+1) w_{2}^{2}-2(n-1) w_{2}+n-1\right\}, \\
& \lim _{\sigma^{2} \rightarrow \infty} e(P T)=1
\end{aligned}
$$

where $w_{1}$ and $w_{2}$ are given in Table 1 .

PROOF. By noting that $\lim _{t \rightarrow \infty} I_{t}(\beta)=1$ for a fixed $\beta$, we have easily the above limits. Since $0<w_{1}, w_{2} \leqq 1$, we have 


$$
2 /(n-1)<\lim _{\sigma^{2}-\infty} e(T 1), \lim _{\sigma^{2} \rightarrow \infty} e(T 2)<(n+1) /(n-1) .
$$

The following equalities among the efficiencies also hold.

COROLLARY 2. For a sufficiently large variance $\sigma^{2}$,

$$
\begin{array}{lll}
e(T 1)>e(P T) & \text { for } & (n-3) /(n+1)<w_{1}<1, \\
e(T 2)>e(P T) & \text { for } & (n-3) /(n+1)<w_{2}<1, \\
e(T 1)>e(T 2) & \text { for } & 0<w_{2}<w_{1}<(n-1) /(n+1) \text { or }(n-1) /(n+1)<w_{1}<w_{2}<1 .
\end{array}
$$

Now we shall make numerical comparisons of the four estimators $T 1, T 2, P T$ and $U$ for the sample $n=7,30,100$ and preliminary conjecture $\left[1 / C_{0}, C_{0}\right]$ with $C_{0}=1.5$, $2.0,2.5$. We take the sample size $n=7$ for a small sample size, $n=30$ for a intermediate sample size and $n=100$ for a large sample size.

\subsection{Comparison of estimators $(n=7)$}

At first we shall examine $e(T 1), e(T 2)$ and $e(P T)$ for $n=7$ in Fig. 1, where the interval of preliminary conjecture is $[0.667,1.5]$. The numerical values of the mean square errors are given in Table 2.

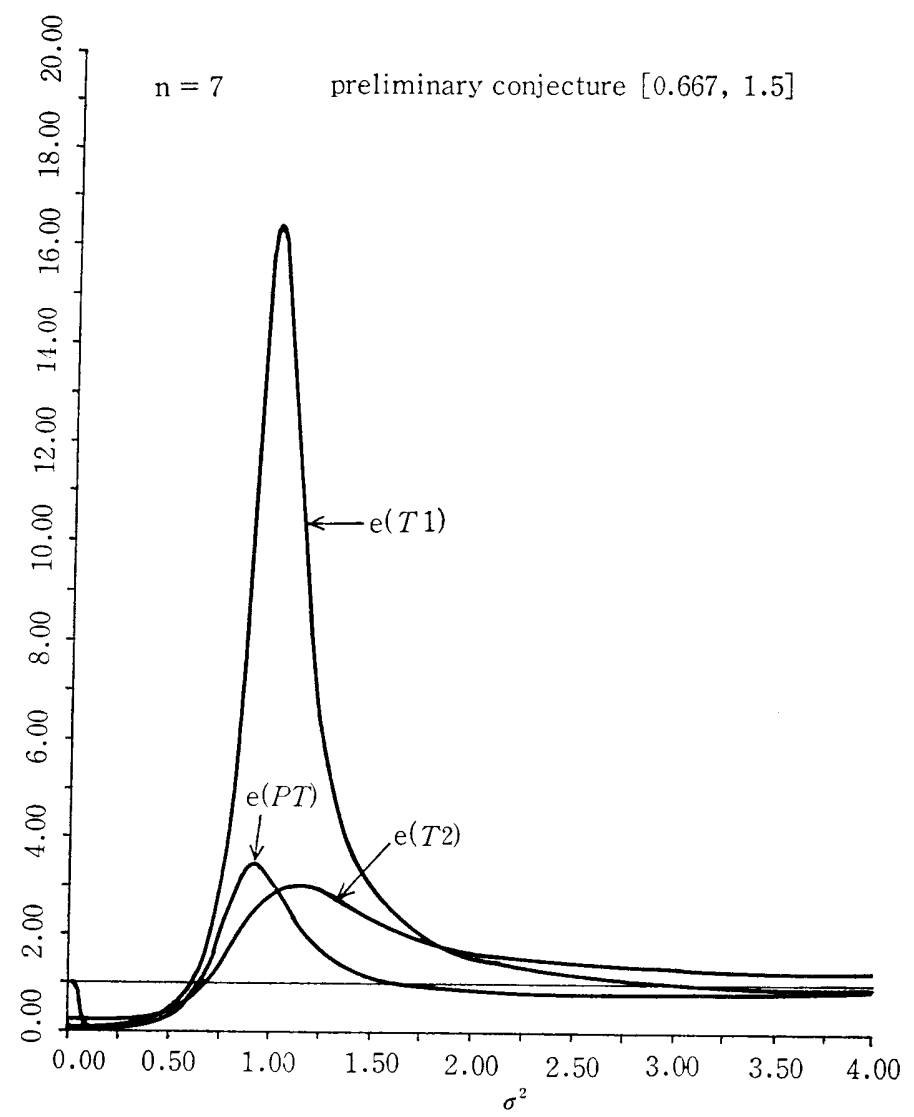

Fig. 1. Efficiencies of $T 1, T 2$ and $P T$ relative to $U$. 
Table 2. Numerical values of the MSE of estimators.

\begin{tabular}{|c|c|c|c|c|}
\hline$\sigma^{2}$ & $\operatorname{MSE}(T 1)$ & $\operatorname{MSE}(T 2)$ & $\operatorname{MSE}(P T)$ & $\operatorname{MSE}(U)$ \\
\hline .1 & .0374 & .0125 & .0459 & .0033 \\
\hline .2 & .1298 & .0501 & .2625 & .0133 \\
\hline .3 & .1926 & .1069 & .3333 & .0300 \\
\hline .4 & .1973 & .1567 & .3010 & .0533 \\
\hline .5 & .1664 & .1768 & .2348 & .0833 \\
\hline .6 & .1223 & .1697 & .1662 & .1200 \\
\hline .667 & .0926 & .1568 & .1273 & .1483 \\
\hline .7 & .0791 & .1495 & .1114 & .1633 \\
\hline .8 & .0450 & .1294 & .0799 & .2133 \\
\hline .9 & .0246 & .1176 & .0774 & .2700 \\
\hline 1.0 & .0202 & .1185 & .1062 & .3333 \\
\hline 1.1 & .0329 & .1334 & .1658 & .4033 \\
\hline 1.2 & .0629 & .1625 & .2539 & .4800 \\
\hline 1.3 & .1099 & .2048 & .3669 & .5633 \\
\hline 1.4 & .1734 & .2593 & .5011 & .6533 \\
\hline 1.5 & .2527 & .3250 & .6524 & .7500 \\
\hline 1.6 & .3469 & .4008 & .8176 & .8533 \\
\hline 1.7 & .4552 & .4858 & .9937 & .9633 \\
\hline 1.8 & .5769 & .5793 & 1.1782 & 1.0800 \\
\hline 1.9 & .7112 & .6807 & 1.3693 & 1.2033 \\
\hline 2.0 & .8574 & .7893 & 1.5655 & 1.3333 \\
\hline 2.1 & 1.0150 & .9048 & 1.7658 & 1.4700 \\
\hline 2.2 & 1. 1834 & 1.0269 & 1. 9694 & 1.6133 \\
\hline 2.3 & 1. 3622 & 1. 1552 & 2. 1760 & 1.7633 \\
\hline 2.4 & 1.5509 & 1.2896 & 2. 3851 & 1.9200 \\
\hline 2.5 & 1.7493 & 1.4299 & 2.5967 & 2.0833 \\
\hline 2.6 & 1. 9570 & 1.5759 & 2.8108 & 2.2533 \\
\hline 2.7 & 2.1737 & 1.7275 & 3.0274 & 2.4300 \\
\hline 2.8 & 2. 3993 & 1.8847 & 3.2467 & 2. 6133 \\
\hline 2.9 & 2.6334 & 2.0474 & 3.4689 & 2.8033 \\
\hline 3.0 & 2.8761 & 2. 2155 & 3.6942 & 3.0000 \\
\hline 3.1 & 3.1270 & 2. 3889 & 3.9227 & 3.2033 \\
\hline 3.2 & 3.3862 & 2.5678 & 4. 1548 & 3.4133 \\
\hline 3.3 & 3.6535 & 2.7519 & 4.3906 & 3.6300 \\
\hline 3.4 & 3.9288 & 2.9413 & 4.6304 & 3.8533 \\
\hline 3.5 & 4. 2121 & 3.1361 & 4.8744 & 4.0833 \\
\hline 3.6 & 4.5033 & 3. 3361 & 5.1227 & 4.3200 \\
\hline 3.7 & 4.8023 & 3.5415 & 5.3757 & 4.5633 \\
\hline 3.8 & 5. 1091 & 3.7521 & 5.6333 & 4.8133 \\
\hline 3.9 & 5.4236 & 3.9680 & 5.8960 & 5.0700 \\
\hline 4.0 & 5.7459 & 4.1891 & 6.1637 & 5.3333 \\
\hline 5.0 & 9.3912 & 6.6923 & 9.1479 & 8.3333 \\
\hline 6.0 & 13.8016 & 9.7287 & 12.7464 & 12.0000 \\
\hline 7.0 & 18.9801 & 13.3027 & 16.9998 & 16.3333 \\
\hline 8.0 & 24.9310 & 17.4172 & 21.9232 & 21.3333 \\
\hline 9.0 & 31.6581 & 22. 0741 & 27.5213 & 27.0000 \\
\hline 10.0 & 39.1640 & 27.2747 & 33.7949 & 33.3333 \\
\hline
\end{tabular}


This shows that $T 1, T 2$ and $P T$ have smaller values of the mean square error than those of $U$ in the neighborhood of $\sigma^{2}=1$. So, $U$ will not be appropriate in our situation. On close investigation from Table 2 it is seen that $e(T 1)>1$ holds on the interval $[0.667,3.2], e(T 2)>1$ holds on the interval $[0.7,10]$ and $e(P T)>1$ holds on the interval $[0.667,1.6]$.

Further, since $e(T 1)>e(P T)$ holds on the interval $[0.1,4]$ and $e(T 1)>e(T 2)$ holds on the interval $[0.5,1.8], T 1$ is more efficient than $P T$ and $T 2$ near one.

As from Table $1, w_{1}$ is smaller than $(n-3) /(n+1)$ and $w_{2}$ is larger than $(n-3) /$ $(n+1), e(T 2)>e(P T)>e(T 1)$ holds for a sufficiently large $\sigma^{2}$.

When the interval of preliminary conjecture are $[0.5,2.0]$ and $[0.4,2.5]$, it is seen that Fig. 2 and Fig. 3 show a similar tendency as Fig. 1.

As from Table 1 , both $w_{1}$ and $w_{2}$ are larger than $(n-3) /(n+1)$ for the intervals of preliminary conjecture $[0.5,2.0]$ and $[0.4,2.5]$ we have $e(T 1)>e(P T)$ and $e(T 2)>$ $e(P T)$ for a sufficiently large $\sigma^{2}$. Also, we have $w_{1}<w_{2}<(n-1) /(n+1)$. Hence, $e(T 2)$ $>e(T 1)>e(P T)$ holds for a sufficiently large $\sigma^{2}$.

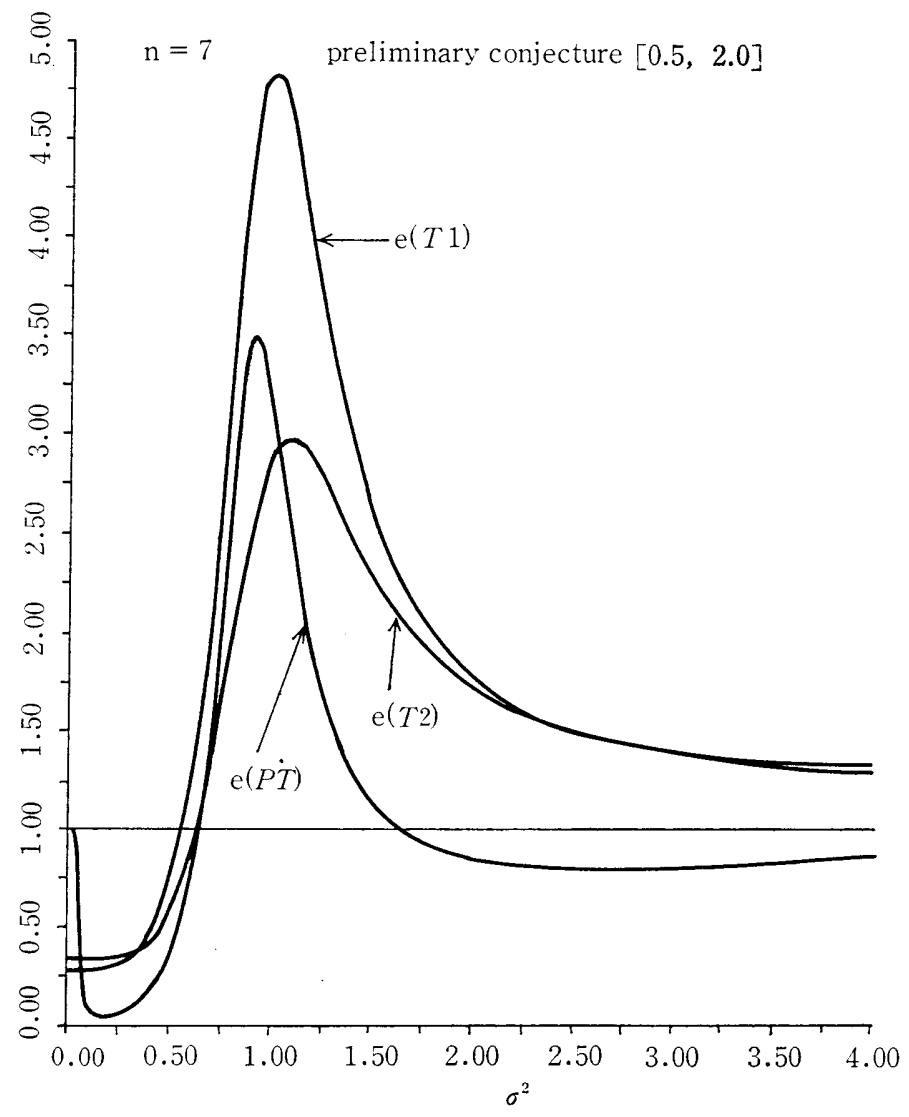

Fig. 2. Efficiencies of $T 1, T 2$ and $P T$ relative to $U$. 


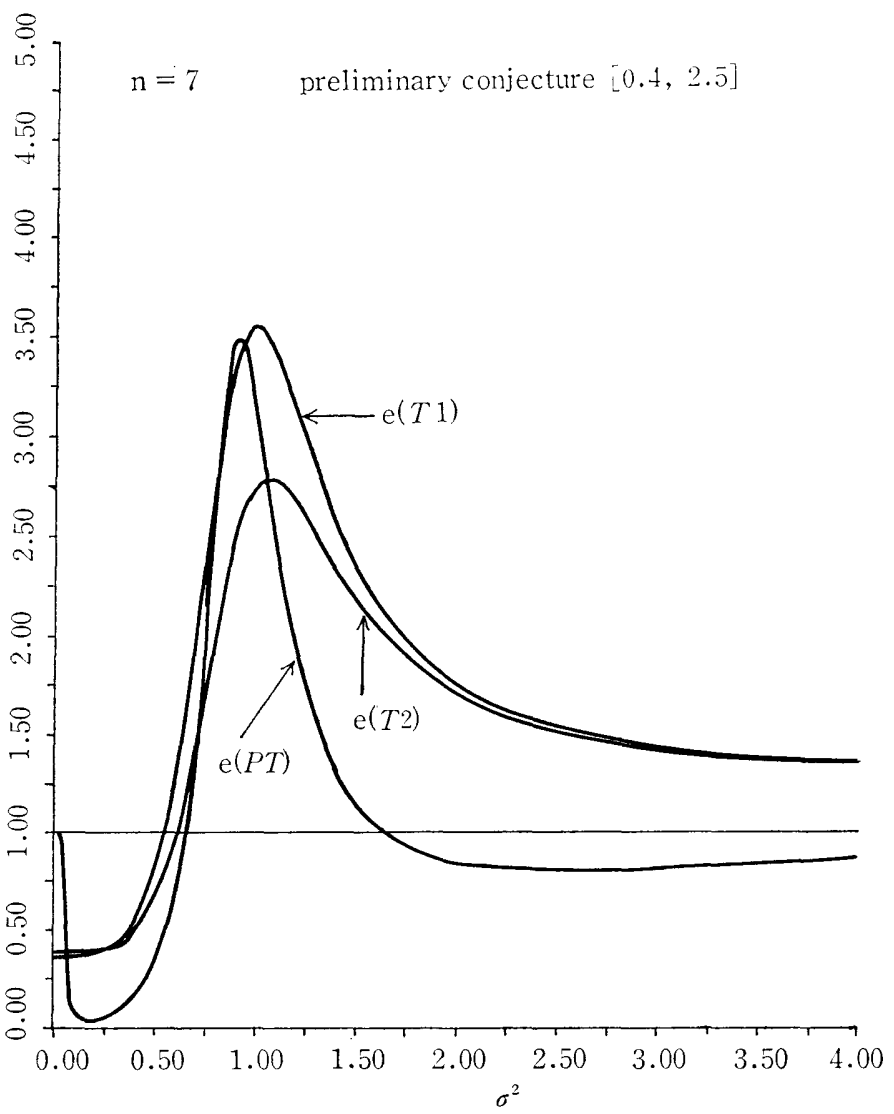

Fig. 3. Efficiencies of $T 1, T 2$ and $P T$ relative to $U$.

\subsection{Comparison of estimators $(n=30)$}

For $n=30$ and the interval of preliminary conjecture $[0.667,1.5]$, Fig. 4 and Table 3 show that $T 1$ and $T 2$ have the smaller mean square error than $P T$ except neighbourhoods of zero and one of $\sigma^{2}$. PT behaves badly for $\sigma^{2}$ near to 0.5 and 1.5 , though it behaves goodly for $\sigma^{2}$ close to one. $T 1$ and $T 2$ behave better than $U$ except for a small $\sigma^{2}$, though they behave a little worse than $U$ for a small $\sigma^{2}$. T2 is only slightly worse than $T 1$ for $\sigma^{2}$ near one and is almost indistinguishable from $T 1$ for $\sigma^{2}$ away from one. Also, for the intervals of preliminary conjecture $[0.5,2.0]$ and $[0.4,2.5]$ we have a similar tendency, and it can be seen that $e(T 2)>e(T 1)>e(P T)$ holds for a sufficiently large $\sigma^{2}$ in each three intervals of preliminary conjecture.

\subsection{Comparison of estimators $(n=100)$}

For $n=100$ and the interval of preliminary conjecture $[0.667,1.5]$, Fig. 5 and Table 4 show that $T 1$ and $T 2$ have the smaller mean square error than $P T$ except neighbourhood of zero and one of $\sigma^{2}$. PT behaves badly for $\sigma^{2}$ near to 0.7 and 1.4 , though it behaves goodly for $\sigma^{2}$ close to one. $T 1$ and $T 2$ behave a little better than $U$ except for a small $\sigma^{2} . e(T 1)$ and $e(T 2)$ are smaller than one for a small $\sigma^{2}$, but the differences 


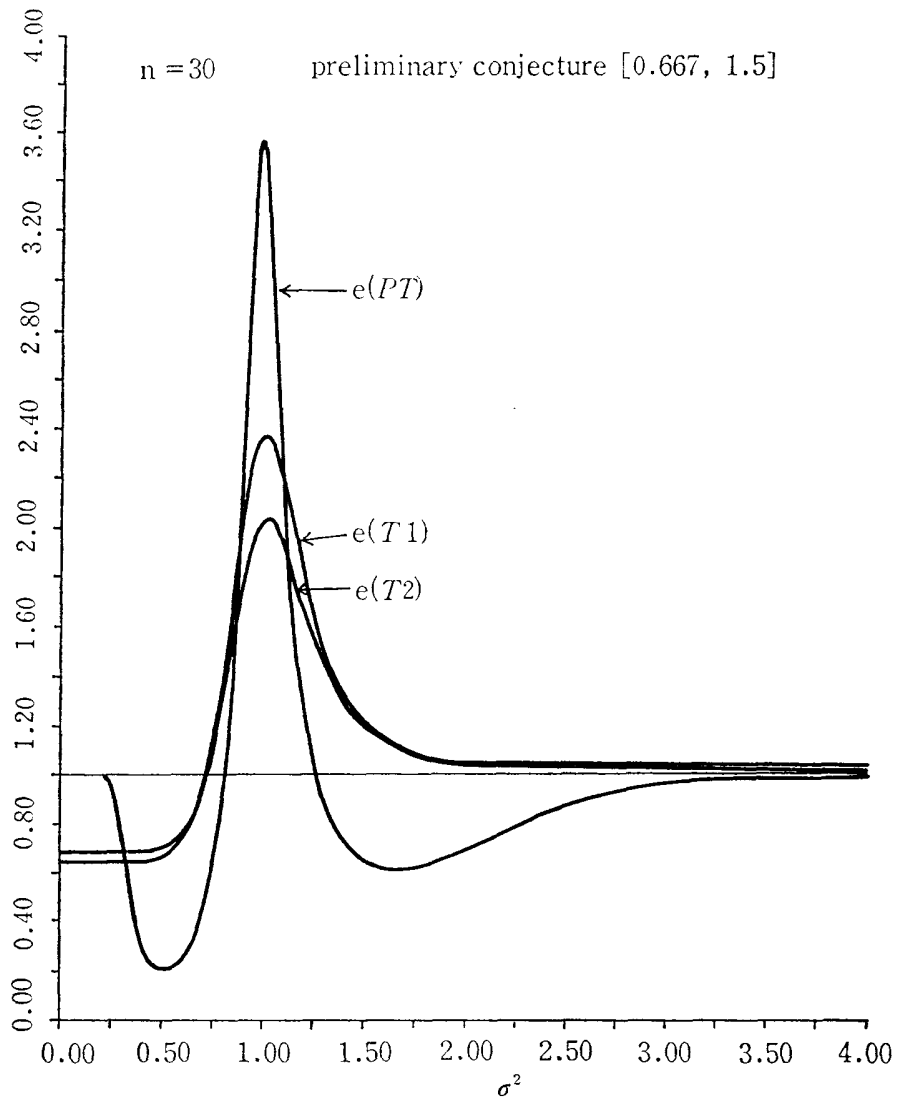

Fig. 4. Efficiencies of $T 1, T 2$ and $P U$ relative to $U$.

are little. The mean square errors of $T 1$ and $T 2$ are almost equal. Also, for the intervals of preliminary conjecture $[0.5,2.0]$ and $[0.4,2.5]$ we have a similar tendency, and it can be seen that $e(T 1)=e(T 2)>e(P T)$ holds for a sufficiently large $\sigma^{2}$ in each three intervals of preliminary conjecture.

In conclusion, for a small or intermediate sample size, we may say that among the estimators $T 1, T 2, P T$ and $U$, the minimax estimator $T 1$ appears to be better than $T 2, P T$ and $U$ with respect to the mean square error criterion, even if our preliminary conjecture, $\sigma_{0}^{2}$, is not very accurate. Though $P T$ behaves goodly for $\sigma^{2}$ close to one, it behaves badly as $\sigma^{2}$ parts from one. $T 1$ and $T 2$ behave goodly except for a small $\sigma^{2}$, but their efficiencies $e(T 1)$ and $e(T 2)$ are not extremely bad for a small $\sigma^{2}$. $T 1$ tends to behave better than $T 2$. Therefore, we should recommend the minimax estimator $T 1$ of variance using preliminary conjecture from the point of view of the mean square error criterion for a small or intermediate sample size.

In order to see the asymptotic properties of $T 1$ and $T 2$, we shall at first take the following approximation to $\operatorname{MSE}[T(w, C)]$ given by (2.7) for a large $n$;

$$
\operatorname{MSE}\left[T(w, C] \doteqdot \sigma^{4}\left[w^{-2}-2 w^{-1}+1\right],\right.
$$


Table 3. Numerical values of the MSE of estimators.

\begin{tabular}{|c|c|c|c|c|}
\hline \multicolumn{2}{|l|}{$n=30$} & \multicolumn{3}{|c|}{ preliminary conjecture $[0.667,1.5]$} \\
\hline$\sigma^{2}$ & $\operatorname{MSE}(T 1)$ & $\operatorname{MSE}(T 2)$ & $\operatorname{MSE}(P T)$ & $\operatorname{MSE}(U)$ \\
\hline .1 & .0011 & .0010 & .0007 & .0007 \\
\hline .2 & .0043 & .0040 & .0028 & .0028 \\
\hline .3 & .0097 & .0091 & .0077 & .0062 \\
\hline .4 & .0172 & .0161 & .0364 & .0110 \\
\hline .5 & .0263 & .0249 & .0864 & .0172 \\
\hline .6 & .0338 & .0330 & .1042 & .0248 \\
\hline .667 & .0355 & .0357 & .0926 & .0307 \\
\hline .7 & .0352 & .0360 & .0827 & .0338 \\
\hline .8 & .0309 & .0335 & .0481 & .0441 \\
\hline .9 & .0271 & .0310 & .0232 & .0559 \\
\hline 1.0 & .0290 & .0337 & .0198 & .0690 \\
\hline 1.1 & .0387 & .0437 & .0407 & .0834 \\
\hline 1.2 & .0553 & .0599 & .0815 & .0993 \\
\hline 1.3 & .0766 & .0806 & .1341 & .1166 \\
\hline 1.4 & .1008 & .1038 & .1895 & .1352 \\
\hline 1.5 & .1266 & .1284 & .2412 & .1552 \\
\hline 1.6 & .1532 & .1537 & .2854 & .1766 \\
\hline 1.7 & .1803 & .1796 & .3213 & .1993 \\
\hline 1.8 & .2078 & .2060 & .3498 & .2234 \\
\hline 1.9 & .2358 & .2330 & .3728 & .2490 \\
\hline 2.0 & .2646 & .2607 & .3924 & .2759 \\
\hline 2.1 & .2941 & .2893 & .4105 & .3041 \\
\hline 2.2 & .3245 & .3189 & .4285 & .3338 \\
\hline 2.3 & .3560 & .3495 & .4476 & .3648 \\
\hline 2.4 & .3885 & .3813 & .4685 & .3972 \\
\hline 2.5 & .4223 & .4142 & .4917 & .4310 \\
\hline 2.6 & .4573 & .4484 & .5174 & .4662 \\
\hline 2.7 & .4935 & .4838 & .5456 & .5028 \\
\hline 2.8 & .5310 & .5205 & .5764 & .5407 \\
\hline 2.9 & .5698 & .5585 & .6096 & .5800 \\
\hline 3.0 & .6099 & .5978 & .6451 & .6207 \\
\hline 3.1 & .6514 & .6384 & .6829 & .6628 \\
\hline 3.2 & .6941 & .6803 & .7228 & .7062 \\
\hline 3.3 & .7383 & .7235 & .7646 & .7510 \\
\hline 3.4 & .7837 & .7680 & .8084 & .7972 \\
\hline 3.5 & .8305 & .8139 & .8540 & .8448 \\
\hline 3.6 & .8787 & .8611 & .9013 & .8938 \\
\hline 3.7 & .9282 & .9096 & .9503 & .9441 \\
\hline 3.8 & .9791 & .9594 & 1.0010 & .9959 \\
\hline 3.9 & 1.0313 & 1.0106 & 1.0532 & 1.0490 \\
\hline 4.0 & 1.0849 & 1.0631 & 1. 1069 & 1.1034 \\
\hline 5.0 & 1.6952 & 1. 6611 & 1.7247 & 1.7241 \\
\hline 6.0 & 2.4410 & 2.3920 & 2.4829 & 2.4828 \\
\hline 7.0 & 3.3225 & 3.2558 & 3.3793 & 3.3793 \\
\hline 8.0 & 4. 3396 & $4.2 \overline{5} 25$ & 4.4138 & 4.4138 \\
\hline 9.0 & 5.4923 & 5. 3821 & 5.5862 & $5 . \overline{3} 362$ \\
\hline 10.0 & 6.7807 & 6.6445 & 6.8966 & 6.8966 \\
\hline
\end{tabular}


Table 4. Numerical values of the MSE of estimators.

\begin{tabular}{|c|c|c|c|c|}
\hline$\sigma^{2}$ & $\operatorname{MSE}(T 1)$ & $\operatorname{MSE}(T 2)$ & $\operatorname{MSE}(P T)$ & $\operatorname{MSE}(U)$ \\
\hline .1 & .0002 & .0002 & .0002 & .0002 \\
\hline .2 & .0009 & .0009 & .0008 & .0008 \\
\hline .3 & .0019 & .0019 & .0018 & .0018 \\
\hline .4 & .0035 & .0035 & .0032 & .0032 \\
\hline .5 & .0054 & .0054 & .0053 & .0051 \\
\hline .6 & .0078 & .0078 & .0149 & .0073 \\
\hline .667 & .0096 & .0096 & .0291 & .0090 \\
\hline .7 & .0105 & .0105 & .0351 & .0099 \\
\hline .8 & .0127 & .0127 & .0336 & .0129 \\
\hline .9 & .0137 & .0137 & .0140 & .0164 \\
\hline 1.0 & .0156 & .0156 & .0054 & .0202 \\
\hline 1.1 & .0200 & .0200 & .0188 & .0244 \\
\hline 1.2 & .0260 & .0260 & .0445 & .0291 \\
\hline 1.3 & .0322 & .0322 & .0655 & .0341 \\
\hline 1.4 & .0383 & .0383 & .0738 & .0396 \\
\hline 1.5 & .0444 & .0444 & .0729 & .0455 \\
\hline 1.6 & .0506 & .0506 & .0698 & .0517 \\
\hline 1.7 & .0572 & .0572 & .0688 & .0584 \\
\hline 1.8 & .0642 & .0642 & .0708 & .0655 \\
\hline 1.9 & .0715 & .0715 & .0755 & .0729 \\
\hline 2.0 & .0792 & .0792 & .0820 & .0808 \\
\hline 2.1 & .0873 & .0873 & .0896 & .0891 \\
\hline 2.2 & .0959 & .0959 & .0980 & .0978 \\
\hline 2.3 & .1048 & .1048 & .1070 & .1069 \\
\hline 2.4 & .1141 & .1141 & .1164 & .1164 \\
\hline 2.5 & .1238 & .1238 & .1263 & .1263 \\
\hline 2.6 & .1339 & .1339 & .1366 & .1366 \\
\hline 2.7 & .1444 & .1444 & .1473 & .1473 \\
\hline 2.8 & .1553 & .1553 & .1584 & .1584 \\
\hline 2.9 & .1666 & .1666 & .1699 & .1699 \\
\hline 3.0 & .1783 & .1783 & .1818 & .1818 \\
\hline 3.1 & .1903 & .1903 & .1941 & .1941 \\
\hline 3.2 & .2028 & .2028 & .2069 & .2069 \\
\hline 3.3 & .2157 & .2157 & .2200 & .2200 \\
\hline 3.4 & .2290 & .2290 & .2335 & .2335 \\
\hline 3.5 & .2426 & .2426 & .2475 & .2475 \\
\hline 3.6 & .2567 & .2567 & .2618 & .2618 \\
\hline 3.7 & .2712 & .2712 & .2766 & .2766 \\
\hline 3.8 & .2860 & .2860 & .2917 & .2917 \\
\hline 3.9 & .3013 & .3013 & .3073 & .3073 \\
\hline 4.0 & .3169 & .3169 & .3232 & .3232 \\
\hline 5.0 & .4952 & .4952 & .5050 & .5050 \\
\hline 6.0 & .7130 & .7130 & .7273 & .7273 \\
\hline 7.0 & .9705 & .9705 & .9899 & .9899 \\
\hline 8.0 & 1.2676 & 1.2676 & 1. 2929 & 1. 2929 \\
\hline 9.0 & 1. 6044 & 1. 6044 & 1.6363 & 1. 6363 \\
\hline 10.0 & 1. 9807 & 1.9807 & 2.0202 & 2.0202 \\
\hline
\end{tabular}




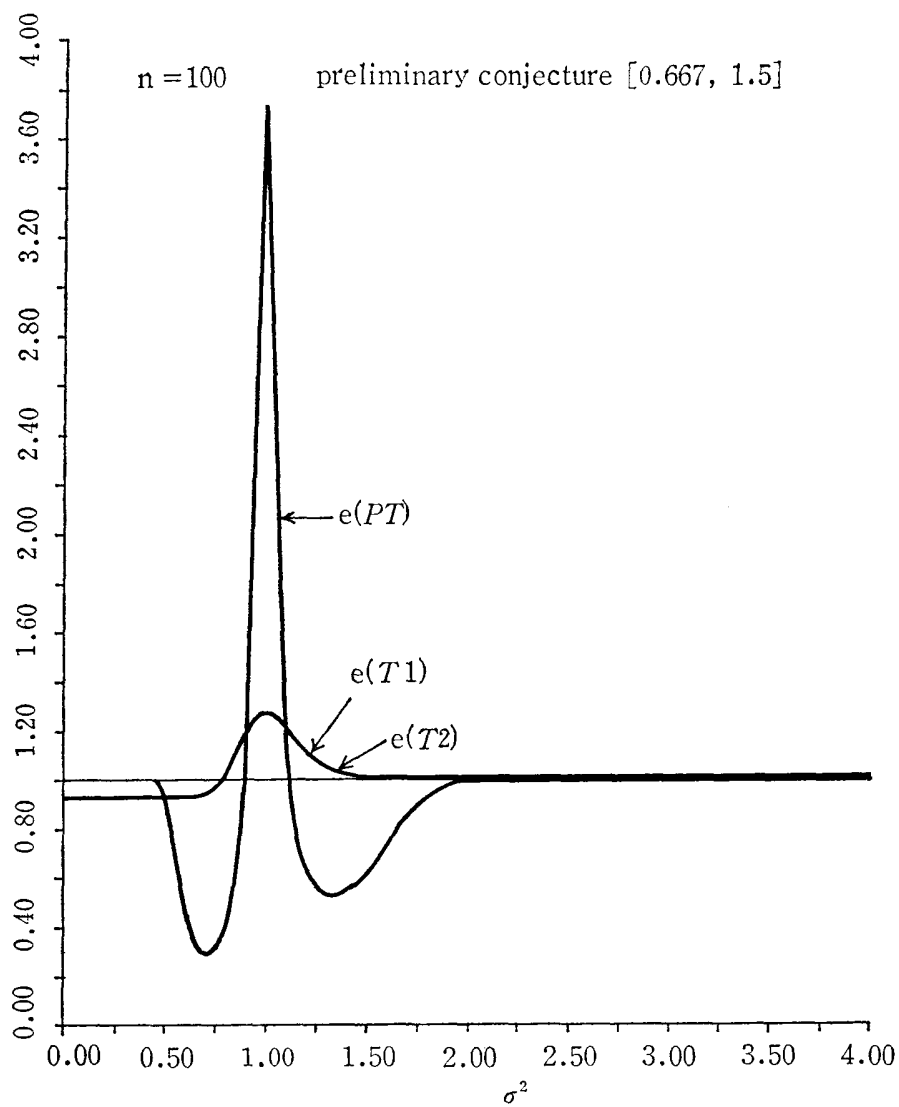

Fig. 5. Efficiencies of $T 1, T 2$ and $P T$ relative to $U$.

which is minimized by $w=1$ for $0<w \leqq 1$. Therefore for a large $n, w_{1}$ is approximately equal to one. For a large $n$, it is seen from Table 1 that $C_{1}$ is approximately equal to one.

Thus, for a large $n$, the estimator $T 1$ is almost equal to the sample unbiased variance $U$. The similar conclusion is extended over to the estimator $T 2$.

The above conclusions will be natural, because the sample information will overcome the prior information as the sample size increases sufficiently large.

\section{Acknowledgements}

The author would like to express his sincere gratitude to Professor Ch. Asano of Kyushu University for his valuable suggestions and constructive comments. The author is also grateful to Professor H. Yamato of Kagoshima University for his advices and encouragements.

\section{References}

[1] Asano, Ch.: Estimation after Preliminary Tests of Significance and their Applications to Biometrical Researches, Bull. Math. Statist., 9 (1960), 1-24. 
[2] Astio, Ch.: Tests due to Pooling Data through Preliminary Tests on Biological Assay, Bull. Math. Statist., 9 (1960), 25-39.

[3] Asayo, Ch.: Some Considerations on the Combination of Estimates from Different Biological Assays, Bull. Math. Statist., 10 (1961), 17-32.

[4] Bancroft, T. A.: On Biases in Estimation due to the Use of Preliminary Tests of Signifcance, Ann. Math. Statist., 15 (1944), 190-204.

[5] BA.Ncroft, T.A. and HAN, Chien-Pai: Inference based on Conditional Specification, a Note and a Bibliography, International Statistical Review, 45 (1977), 117-127.

[6] BenNett, B. M.: Estimation of Means on the Basis of Preliminary Tests of Significance, Ann. Inst. Statist. Math., 4 (1952), 31-43.

[7] Berger, J.O.: Statistical Decision Theory and Baysian Analysis, Springer-Verlag, New York, (1985).

[8] Hirano, K.: Estimation Procedures based on Preliminary Tests, Shrinkage Technique and Information Criterion, Ann. Inst. Statist. Math., 29A (1977), 21-34.

[9] Hiravo, K.: On Level of Significance of the Preliminary Test in Pooling Means, Ann. Inst. Statist. Math., 30A (1978), 1-8.

[10] HiRano, K.: A Note on the Level of Significance of the Preliminary Test in Pooling Variances, J. Japan Statist. Soc., 8 (1978), 71-75.

[11] Hirano, K.: On the Test Estimators for Estimating Variance of a Normal Distribution, Recent Developments in Statistical Inference and Data Analysis, (ed. Matusita, K.), NorthHolland Publishing Company, (1980), 105-115.

[12] Kitagawa, T.: Successive Process of Statistical Inferences, Mem. Fac. Sci. Kyushu Univ., A. 5 (1950), 139-180.

[13] Kudo, H.: On Partial Prior Information and the Property of Parametric Sufficiency, Proc. Fifth Berkeley Symp. Math. Statist. Prob., 1 (1967), 251-265.

[14] Ohtani, K. and Toyoda, T.: Minimax Regret Critical Values for a Preliminary Test in Pooling Variances, J. Japan Statist. Soc., 8 (1978), 15-20.

[15] Toyoda, T. and Wallace, T.D.: Estimation of Variance after a Preliminary Test of Homogeneity and Optimal Levels of Significance for, the Pre-test, J. Econometrics, 3 (1975), 395-404.

[16] Yamauti, Z.: Statistical Tables and Formulas with Computer Applications JSA-1972, Japanese Standards Association, (1972)

Received September 13, 1988

Communicated by Ch. Asano 